\title{
Psychomotor vigilance task- objective sleep disorder screening tool for Indian population
}

\author{
Vanita C Ramrakhiyani, Abhijit G Deshpande \\ Prajakta A Deshpande, Prasad C Karnik
}

International Institute of Sleep Sciences, Bungalow 10, MHADA Colony, Off Eastern Express Highway, Mulund (E), Mumbai 400081 , Maharashtra

DOI No : 10.5958/i.0974-0155.7.4.023

\begin{abstract}
Introduction / Objective: The primary objective of the current pilot observational study was to establish feasibility and acceptability of sleep disorder screening tools, viz, Epworth Sleepiness Scale (ESS) and Psychomotor Vigilance Task (PVT) in Indian Population. The study also aimed to compare the sensitivity and specificity of both the screening tests. The outcome of the study will provide the threshold values for PVT to determine positive and negative diagnosis.

Method: It was a community based study in clinical settings. Patients visiting sleep clinic during period of year August 2011 to year September 2012 formed the study population. The sample population included 66 patients; 49 males and 17 females with mean age of 45 (age range 14-75). All subjects administered ESS as well as 10 minute PVT followed by confirmatory tools such as Nocturnal Polysomnogram, MSLT and Actigraphy. ESS score > 10 was considered positive. The presence of any of intrinsic or extrinsic sleep disorder is considered a positive diagnosis. Bayesian theorem was applied to determine sensitivity and specificity of tests administered.

Results: Sensitivity of PVT was found to be high as $90.16 \%$ as compared to that of ESS as $39.3 \%$. However the specificity for both the tests was found to be equal at $100 \%$. Among PVT measurements, number of lapses and average reaction time were found to be co-relating with a sleep disorder diagnosis.

Conclusion: Individuals having low ESS score were diagnosed with sleep problems which were well predicted in their PVT results. For Indian population, PVT assessment is more sensitive screening tool for sleep disorders as compared to ESS. Although, when administered together, chances of missing any sleep problem are minimised. It was a pilot study and needs to be further validated with larger population.
\end{abstract}

Address for correspondence

\section{Vanita C Ramrakhiyani}

International Institute of Sleep Sciences,

Bungalow 10, MHADA Colony,

Off Eastern Express Highway,

Mulund (E), Mumbai 400 081, Maharashtra

Email: vanitar@iiss.asia

\section{Introduction}

S leep is a necessary part of human functioning. Sleep disorders impair quality of life and thereby poses several health related problems ${ }^{19}$. The disease burden for sleep disorders is huge in Indian population. Commonly found sleep disorders are insomnia, Obstructive sleep apnea, Hypersomnia, Restless leg syndrome and Shift work disorder ${ }^{17,19,22 .}$ 
A questionnaire based sleep survey conducted in 2009, revealed that 93 per cent of Indians are not sleeping enough and 34 per cent are at risk of Obstructive Sleep Apnea (OSA), which can lead to weight gain and even serious situations such as worsening of the heart conditions ${ }^{1}$. Sleep Medicine being a recent field in Indian sub-continent, the availability of data is sparse. Most of these studies are performed on urban population and are based on subjective questionnaires ${ }^{19}$. Current protocol being followed at sleep disorder clinics in India includes use of subjective sleepiness scales such as Epworth Sleepiness Scale (or its modified versions) to screen the subjects. 'Our observations reflect that because of social factors, there is denial of symptom of EDS in symptomatic as well as asymptomatic people. Modification of ESS for Indian population has been studied but no significant difference has been reported ${ }^{19}$. Reporting heterogeneity in self reports on health has been studied in Indian population. The study revealed an underestimation of health related issues in low-income population and overestimation among high income group $^{24}$.

There is a need to objectively screen the symptom of sleepiness. Psychomotor vigilance task is a proven

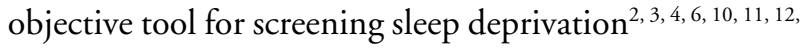
${ }^{23}$. PVT is a sustained-attention, reaction-timed task. It is freely available software based test and can be taken by individuals with normal intellectual skills. It captures the neurocognitive effects of sleep loss on wake state stability as reflected in sustained attention ${ }^{12}$. Research indicated increased sleepiness correlates with deteriorated alertness, slower problem-solving, declined psycho-motor skills, and increased rate of false responding $2,3,4,6,12$. PVT sensitivity has been proven in acute sleep loss, chronic partial sleep deprivation, effect on jet lag \& shift work syndrome ${ }^{2}$. PVT sensitivity has been extensively studied on sleep disordered breathing ${ }^{10}$

The study aimed to compare subjective and objective sleepiness scores for patients visiting sleep clinic and thereby established a highly sensitive and reliable screening tool. The outcome of the study would determine the cut-off values for PVT parameters to be used in clinical set up.

\section{Methodology}

Study Population: The present study was conducted at International Institute of Sleep Sciences, Mulund,
Mumbai; over a period from August 2011 to September 2012. The study population comprises of 68 patients; 49 males and 17 females (Table-1). The study subjects were screened with the help of detailed History-Physicals. The subjects were consecutively selected from age group of 14-75 of either gender referred for sleep study at clinic. The tests were performed between 9:00-11:00 am to negate the effect of circadian dip.

Tests: All subjects were administered ESS as well as 10 minute PVT followed by Nocturnal Polysomnogram or Actigraphy or MSLT as per the history-physical data.

In ESS questionnaire, a patient is asked to rate the likelihood that he or she would fall asleep in given situations (Figure 1). The patient rates each situation on a scale of 0 to 3 , with 3 being a high chance of dozing or falling asleep, and 0 if they would never doze or sleep in the given situation. The scores are then added up, and the Epworth Sleepiness Scale is revealed. With as many as 3 points possible in each situation, the scale reaches a high of 24. The patient and physician can compare the patient's Epworth Sleepiness Score with the following

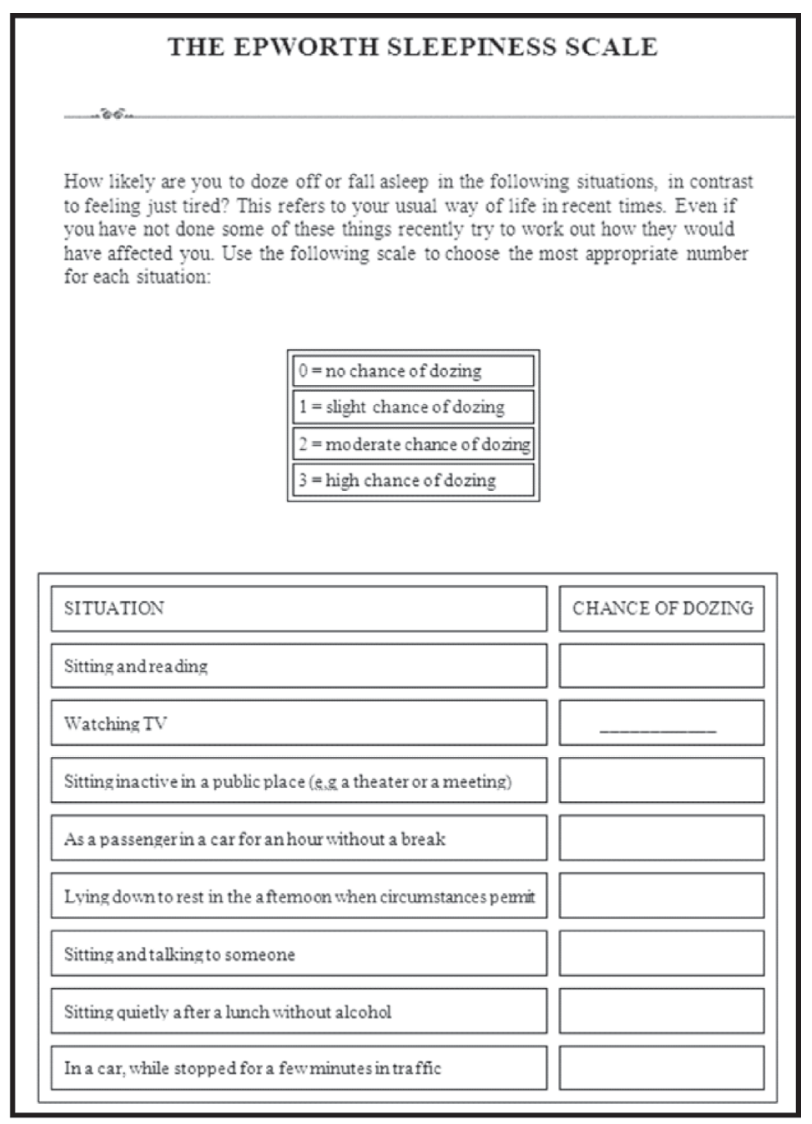

Figure 1: Epworth Sleepiness Scale 
scale:

0-9Normal Sleepiness

\section{0-24 Excessively Sleepy}

The PEBL software version 0.11(Shane Mueller August 2010) was utilized for PVT. The subject is seated comfortably. The subject is asked to respond as soon as possible to red circle appearing in the center of the screen by pressing "Space Bar" on computer keypad. The red circle keeps on coming in the interval of 2-10 seconds. The reaction time is calculated in milliseconds. The task duration is 10 minutes (Figure - 2).
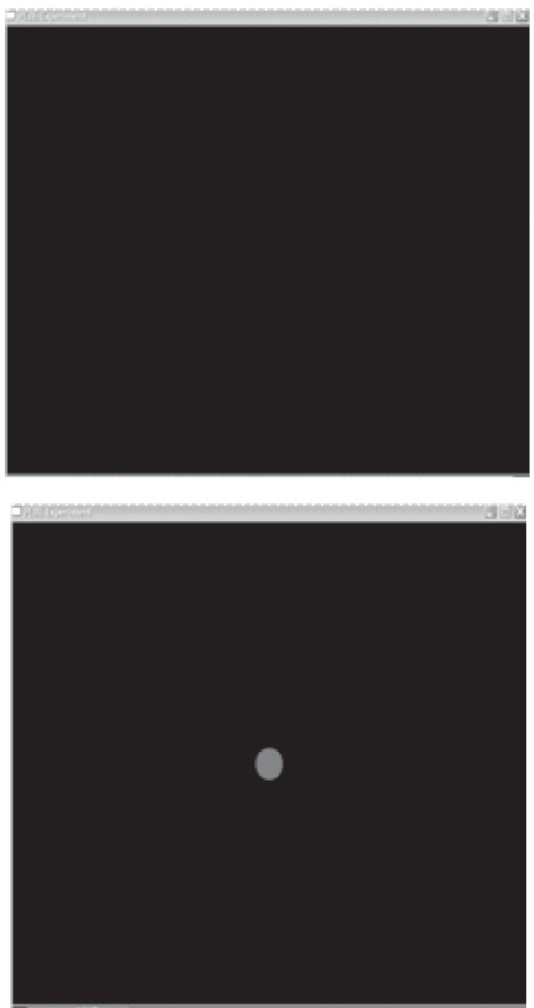

Figure 2: PVT

\section{Scores for PVT}

Average Reaction time, No. of lapses/Errors of Omission (RT > 500 milliseconds)

No. of sleep responses (RT > 30 secs) and No. of too fast responses/ Errors of Commission

Polysomnography studies were conducted and scored as recommended by the American Academy of Sleep Medicine. All EEG electrodes have been placed according to the "International 10-20 System".

\section{Montage Utilized}

1) EEG-C4 referenced to $\mathrm{A} 1$

2) EEG-O2 referenced to $\mathrm{A} 1$

3) REOG- referenced to $\mathrm{A} 1$

4) LEOG- referenced to A2

5) $\mathrm{EEG}-\mathrm{C} 3$ referenced to $\mathrm{A} 2$

6) $\mathrm{EEG}$ - O1 referenced to A2

7) Chin EMG

8) EKG

9) Left and Right Anterior Tibialis

10) Snore Microphone

11) Nasal and Oral Airflow - Thermocouple

12) Thoracic respiratory effort

13)Abdominal respiratory effort

14) Pulse Oximetry - finger probe

The presence of any of intrinsic or extrinsic sleep disorder is considered a positive diagnosis. In order to eliminate any possible transient improvement in PVT brought about by use of CPAP for titration purposes in split night studies, all subjects underwent PVT as well as ESS before the sleep study.

Statistical Analysis: The Null hypothesis was "diagnosis with PVT was equal to diagnosis with ESS". Paired " $\mathrm{t}$ " test was applied as same population is being compared as well as sample size was $>30$. Bayesian theorem was utilised to determine sensitivity and specificity of tests administered.

\section{Results}

Records of 66 patients from sleep disorder clinic were analyzed. Mean age of the subjects was 45 years (range: 14-75). The demographic characteristics of study population are depicted in Table 1 . All the subjects performed ESS as well as 10 minute PVT without any difficulty. The study established the feasibility and acceptability of both screening tools for Indian population (Table-2).

The frequency of subjects scoring $>10$ in ESS was found $36.36 \%$. PVT outcome metrics used were: number of lapses, number of false starts, average reaction 
time. The correlation between PVT parameters and sleep disorder diagnosis is clearly evident in graph 1,2 and 3

Table 1: Demographic characteristic of study population

\begin{tabular}{|l|}
\hline Variables \\
$\mathrm{N}=66$ \\
Mean age (range) years 45(14-75) \\
Male; Female 49;17 \\
Residence \\
Urban 66 \\
Educational status \\
School educated 12 \\
College educated 54 \\
\hline
\end{tabular}

Table 2: Acceptability of screening tools

\begin{tabular}{|l|l|l|}
\hline & Given to & Acceptability \\
\hline ESS & 68 & $100 \%$ \\
\hline PVT & 68 & $100 \%$ \\
\hline
\end{tabular}

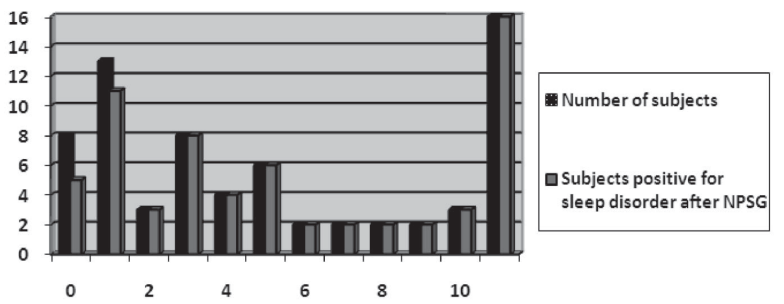

Graph 1: correlation between number of lapses (Xaxis) and positivity of Sleep Disorder. The threshold value is $>2$ lapses i.e. error of omission

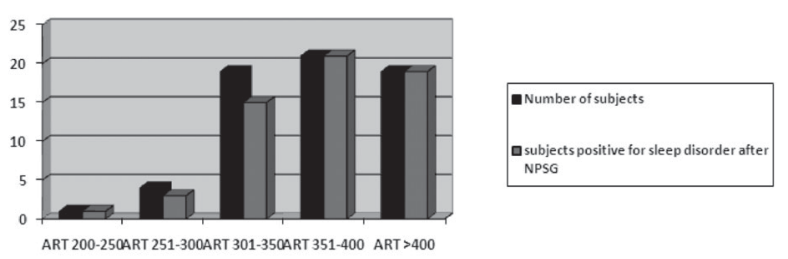

Graph 2: correlation between Average Reaction Time (X-axis) and positivity of Sleep Disorder. The threshold value is $>350$ milliseconds.

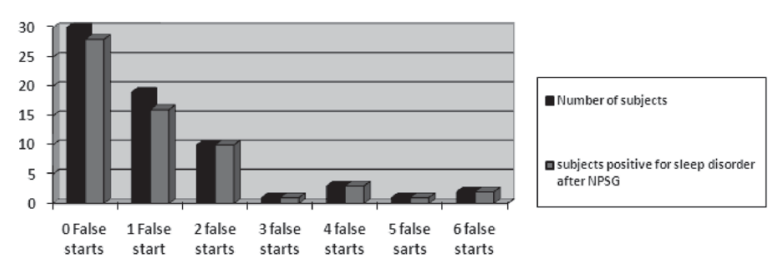

Graph 3: correlation between number of error of commission (X-axis) and positivity of Sleep Disorder.

The threshold value is $>2$ error of commission
Table 3: Screening results for ESS

\begin{tabular}{|l|l|l|l|}
\hline \multirow{2}{*}{ Screening Results for ESS } & \multicolumn{2}{|l|}{$\begin{array}{l}\text { True Characteristics } \\
\text { in Population }\end{array}$} & Total \\
\cline { 2 - 4 } & Disorder & No Disorder & \\
\hline Positive & 24 & 0 & 24 \\
\hline Negative & 37 & 5 & 42 \\
\hline Total & 61 & 5 & 66 \\
\hline
\end{tabular}

Table 4: Screening results for PVT

\begin{tabular}{|l|l|l|l|}
\hline \multirow{2}{*}{ Screening Results for ESS } & \multicolumn{2}{l|}{$\begin{array}{l}\text { True Characteristics } \\
\text { in Population }\end{array}$} & Total \\
\cline { 2 - 4 } & Disorder & No Disorder & \\
\hline $\begin{array}{l}\text { Positive( }>2 \text { errors/ } \\
\text { Average ART }>350 \mathrm{~ms})\end{array}$ & 55 & 0 & 55 \\
\hline Negative & 6 & 5 & 11 \\
\hline Total & 61 & 5 & 66 \\
\hline
\end{tabular}

Table 5: Comparison of diagnostic parameters for ESS and PVT

\begin{tabular}{|l|l|l|}
\hline & ESS & PVT \\
\hline Sensitivity & $39.3 \%$ & $90.16 \%$ \\
\hline Specificity & $100 \%$ & $100 \%$ \\
\hline Positive Predictive Value & 100 & 100 \\
\hline Negative Predictive Value & 12 & 45 \\
\hline
\end{tabular}

Table 6: " $\mathrm{t}$ " test scores for ESS and PVT

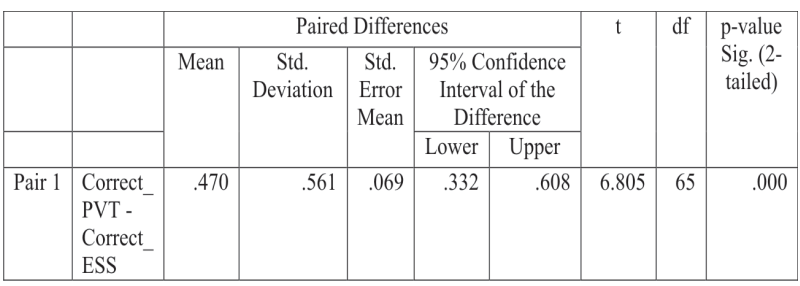

Table 7: Prevalence of Common Sleep Disorders in study population

\begin{tabular}{|l|l|l|}
\hline Subjects positive for Sleep disorder & Number & Percent \\
\hline Obstructive Sleep Apnea & 31 & $47 \%$ \\
\hline REM Related Sleep Apnea & 4 & $6 \%$ \\
\hline Insomnia causing Sleep Deprivation & 14 & $21.12 \%$ \\
\hline Narcolepsy & 3 & $4.5 \%$ \\
\hline Upper Airways Resistance Syndrome & 4 & $6 \%$ \\
\hline $\begin{array}{l}\text { Delayed Phase Syndrome causing Sleep } \\
\text { Deprivation }\end{array}$ & 2 & $3.03 \%$ \\
\hline
\end{tabular}


respectively. The threshold values to be used clinically for PVT were found to be $>2$ Errors and/ ART $>350$ milliseconds. Screening results for both the tests are given in table no 3 and 4 respectively. The sensitivity of ESS and PVT were $39.3 \%$ and $90.16 \%$ respectively. The specificity for both the tests was found to be $100 \%$.

Since the p-value was found to be 0.00 (which is $<0.05$ with $95 \%$ Confidence Interval), null hypothesis was rejected.

The prevalence of common sleep disorders in this clinical population is tabulated in Table 7.

\section{Discussion}

Low perception of sleep disorders was observed in Indian population. Despite doing few modifications in subjective questionnaires such as ESS, as per Indian context, the scores were not correlated with diagnosis. The frequency of unanswered questions in ESS and modified ESS was

\begin{tabular}{|l|l|}
\hline Study & PVT outcome metrics \\
\hline In-Soo Lee et.al; 2010 & $\begin{array}{l}\text { Count of lapses, Average } \\
\text { Reaction Time }\end{array}$ \\
\hline Glenn Gunzulmenn et al; 2008 & $\begin{array}{l}\text { False starts, Alert responses, } \\
\text { Lapses and sleep attacks }\end{array}$ \\
\hline Adreinne Tucker; 2009 & $\begin{array}{l}\text { False starts, Lapses and Mean } \\
\text { Reaction Time }\end{array}$ \\
\hline David Dinges; 1998 & Number of Lapses \\
\hline Basner and Dinges; 2011 & $\begin{array}{l}\text { Number of lapses, mean RT, } \\
\text { mean 1/RT, fastest 10\% RT, } \\
\text { median RT, slowest 10\% RT, } \\
\text { slowest 10\% 1/RT, number of } \\
\text { false starts, fastest 10\% 1/RT } \\
\text { and lapse probability }\end{array}$ \\
\hline
\end{tabular}

found to be as high as $23.9 \%$ and $45.3 \%$ respectively. Therefore, Critical evaluation of the prevalent subjective screening questionnaires for sleep disorders is warranted in the Indian context ${ }^{19}$.

The current study investigated the feasibility and acceptability of sleep disorder screening tools viz, ESS and PVT. ESS has its known advantage of being a short, simple form that the patient can complete without assistance ${ }^{5,8}$. The present findings suggest that PVT was equally feasible and acceptable. Both the tests were economical for clinical settings.

The PVT parameters reported in earlier studies are tabulated below.
In a previous study, number of lapses and 1/Average Reaction Time are considered to be superior PVT outcome metric to be used as criterion in sleep deprivation experiment ${ }^{2}$. The current study took into consideration basic PVT parameters viz, number of lapses, mean reaction time and false starts which can be easily utilized in clinical setting.

The study results indicate that the number of lapses increases exponentially with confirmed sleep disorder diagnosis. The threshold values for the same are 2 . Our findings of a significant relationship between reduced PVT performance and sleep disorder diagnosis was expected and were consistent with previous studies.

The reported prevalence of common sleep disorders in Indian population, such as Insomnia, Obstructive Sleep Apnea (OSA) and Restless Leg Syndrome (RLS) is $18.6 \%, 9.3 \%$ and $2.9 \%$ respectively ${ }^{19}$. In the current study, the prevalence rates for OSA and Insomnia in clinical population were found to be $47 \%$ and $21.12 \%$ respectively.

The present study was a pilot step with low sample size. The study results are limited to clinical population only; larger studies are needed to validate these findings further in general population. The significance of this study lied in the fact that a highly sensitive screening tool such as PVT, at the clinician's end would reduce the underdiagnosis and/or misdiagnosis of sleep disorder cases. The applicability of combination of these tests can be studied in future. Despite its limitations, the present study has created a platform for effective screening of sleep disorders in Indian population.

\section{References}

1. "Waking up to sleep therapy"; Express Healthcare; June 2010.

2. Adrienne $M$ Tucker, Robert $C$ Basner, Yaakov Stern, Brian C. Ratkin; "The Variable Response-Stimulus Interval Effect and sleep deprivation: An unexplored aspect of Psychomotor Vigilance Task Performance."; Sleep; Vol. 32, No. 10, July 2009, pg no. 1393-1395.

3. Clare Anderson, et.al;" PVT lapses differ according to eyes open, closed or looking away"; Sleep, vol 33, no. 2, 2010, pg no.197-204.

4. Daniel A Cohen et.al.;"Uncovering residual effects of chronic sleep loss on human performance"; Sci Transl med, vol 2 no. 14, 2010, pg no.1-15.

5. Daniel J. Buysse, M.D; Martica L. Hall, Ph.D.; Patrick J. Strollo, M.D.; Thomas W. , Ph.D.; Jane Owens, Ph.D.; Laisze Lee, M.S.; Steven E. Reis, M.D.; Karen A. Matthews, PhD;

Indian Journal of Sleep Medicine (IJSM), Vol. 7, No. 4, 2012 
"Relationships Between the Pittsburgh Sleep Quality Index (PSQI), Epworth Sleepiness Scale (ESS), and Clinical/ Polysomnographic Measures in a Community Sample"; Journal of Clinical Sleep Medicine, Vol. 4, No. 6, 2008

6. David R. Thorne et.al; "The Walter palm-held psychomotor vigilance test"; Behavior Research methods, vol 37, no 1 , 2005, pg no 111-118.

7. G Stores; "Clinical diagnosis and misdiagnosis of sleep disorders"; Journal of Neurol Neurosurg Psychiatry 2007; 78; 1293-1297.

8. Hanish Sharma et.al;" Pattern \& correlates of neurocognitive dysfunction in Asian Indian adults with severe obstructive sleep apnea"; Indian journal of Med Res; vol 132, 2010, pg no. 409-414.

9. Hans P.A. et.al;" The cumulative cost of additional wakefulness: Dose-response effects on neurobehavioral functions and sleep physiology from chronic sleep restriction and total sleep deprivation"; Sleep vol 26 no. 2, 2003,pg no. 117-126.

10. Hyon Kim, David F Dinges, Terry Young;"Sleep-Disordered breathing and Psychomotor vigilance in a community-based sample"; Sleep, Vol.30,No. 10, 200, pg no.1309-1316.

11. In-Soo Lee et.al; "Number of lapses during the psychomotor Vigilance Task as an objective measure of fatigue"; Journal of clinical sleep medicine; vol 6, no. 2, 2010, pg no.163-168.

12. Jillian Dorrian, Naomi L. Rogers and David F. Dinges; "Psychomotor Vigilance Performance: Neurocognitive Assay Sensitive to Sleep Loss"; University of Pennsylvania School of Medicine, Philadelphia, Pennsylvania, USA.

13. Mark S Aloia; "Neuropsychological consequences of obstructive sleep apnea-considerations for treatment"; Business briefing: US Respiratory Care, 2005, pg no.1-5.

14. Matthew D. Kafta; "Effects of Sleep Deprivation"; South Dakota School of Mines \& Technology, pg no. 73-79.
15. Murray W. Johns; "A New Method for Measuring Daytime Sleepiness: The Epworth Sleepiness Scale"; Sleep, $14(6): 540-545$.

16. Nancy Lynn Grugle; "Understanding the Effects of Sleep Deprivation on Executive Function, Complex Task Performance and Situation Awareness"; PhD Dissertation, Virginia Polytechnic Institute and State University, Virginia, 2005.

17. Nilesh Shah, Abha Bang, Aparna Bhagat; "Indian research on sleep disorders"; Indian Journal of Psychiatry, 52, supplement, January 2010.

18. R. Nisha Aurora et al; "Correlating subjective and objective sleepiness: Revisiting the association using survival analysis"; Sleep, vol 34, No. 12, 2011.

19. Samhita Panda et.al.; "Sleep-related disorders among a healthy population in South India"; Neurology India; Vol 60, Issue 1, Feb 2012.

20. Sarah Ledoux; "The Effects of Sleep Deprivation on Brain and Behavior"; Biology 202, 2002 third web report.

21. Sarah Nath Zallek, M.D; Rachel Redenius; Holly Fisk, B.S; Carli Murphy, B.S; Erin O'Nei; "A Single Question as a Sleepiness Screening Tool"; Journal of Clinical Sleep Medicine, Vol. 4, No. 2, 2008.

22. Surendra K. Sharma \& Gautam Ahluwalia: "Epidemiology of adult obstructive sleep apnoea syndrome in India"; Indian $J$ Med Res 131, February 2010, pp 171-175.

23. Sylvia Loh et.al." The validity of psychomotor vigilance tasks of less than 10-minute duration", Behaviour research methods, instruments \& computers; Vol 36 no. 2, 2004, pg no. 339-346

24. Teresa Bago et.al; "Does reporting heterogeneity bias the measurement of health disparities?" Tinbergen Institute Discussion paper, TI 2006-033/3.

25. Zarir F. Udwadia, Amita V. Doshi, Sharmila G. Lonkar, and Chandrajeet I. Singh; "Prevalence of sleep-disordered breathing and sleep apnea in middle-aged urban Indian men"; American Journal of Respir Crit Care Med; vol 169 pp 168-173, 2004. 